\title{
Microscopic and Macroscopic Properties of Carbohydrate Solutions in the Ionic Liquid 1-Ethyl-3-methyl-imidazolium Acetate
}

\author{
Michael E. Ries, ${ }^{*}{ }^{\dagger}$ Asanah Radhi, ${ }^{\dagger}$ Stephen M. Green, ${ }^{\dagger}{ }^{\oplus}$ Jamie Moffat, ${ }^{\ddagger}$ and Tatiana Budtova ${ }^{\S}$ \\ ${ }^{\dagger}$ Soft Matter Physics Research Group, School of Physics and Astronomy, University of Leeds, Leeds LS2 9JT. U.K. \\ ${ }^{\ddagger}$ Innovia Films R\&D Centre, West Road, Wigton, Cumbria CA7 9XX, U.K. \\ ${ }^{\S}$ MINES ParisTech, PSL Research University, Center for Materials Forming (CEMEF), UMR CNRS 7635, CS 10207, 06904 \\ Sophia Antipolis, France
}

Supporting Information

ABSTRACT: Solutions of glucose, cellobiose, and microcrystalline cellulose in the ionic liquid 1-ethyl-3-methylimidazolium acetate $([\mathrm{C} 2 \mathrm{mim}][\mathrm{OAc}])$ have been examined using low-field $(20 \mathrm{MHz})$ NMR relaxometry and rheology. The spin-lattice $\left(T_{1}\right)$ and spin-spin $\left(T_{2}\right)$ relaxation times have been determined from 30 to $70{ }^{\circ} \mathrm{C}$ inclusive, for a range of concentrations (0-15 wt \%) of each carbohydrate in $[\mathrm{C} 2 \mathrm{mim}][\mathrm{OAc}]$. The zero shear rate viscosities for the same samples across the same temperature range were studied. The viscosity, NMR relaxometry, and previously published diffusion data were all analyzed together through the Debye-Stokes-Einstein equations. Microscopically, these systems behave as an "ideal mixture" of free ions and ions associated with the carbohydrate molecules. The molar ratio of carbohydrate $\mathrm{OH}$ groups to ionic liquid molecules, $\alpha$, is the key parameter in determining the NMR relaxometry and hence the local microscopic environment of the ions. NMR relaxometry data are found to follow an Arrhenius type behavior, and the difference in rotational activation energy between free and associated ions is determined at $6.2 \pm 0.5 \mathrm{~kJ} / \mathrm{mol}$.

\section{INTRODUCTION}

In 1914, Walden defined an ionic liquid (IL) to be a salt, which has a liquid state below $100{ }^{\circ} \mathrm{C}$ at atmospheric pressure. ${ }^{1}$ Since then, and particularly more recently, there has been much research into using ILs as "green" solvents. ${ }^{2-7}$ This is because their properties can include low to negligible vapor pressure, high thermal stability, low flammability, and the ability to dissolve natural compounds such as polysaccharides. ${ }^{8}$ In 1934, Graenacher ${ }^{9}$ obtained a patent for, amongst other things, dissolving cellulose with molten $\mathrm{N}$-ethylpyridinium chloride. This salt has a melting point at $118{ }^{\circ} \mathrm{C}$ and therefore does not fall under the prior Walden definition of an IL. Swatloski et al. in 2002 published on the use of imidazolium-based ILs to dissolve cellulose. ${ }^{10}$ In this work, the authors measured the solubility of cellulose in a variety of salts and found that 1butyl-3-methyl-imidazolium chloride $[\mathrm{C} 4 \mathrm{mim}][\mathrm{Cl}]$ dissolved the greatest amount of cellulose, up to $25 \mathrm{wt} \%$ upon microwave heating.

It is often said that cellulose is the world's most abundant biopolymer, and it is indeed one of the most studied with the term "cellulose" dating back to 1839 and the pioneering work of Payen. ${ }^{11}$ As cellulose does not melt, the processing of cellulose requires dissolution and/or derivatization, with objects such as fibers and films being formed. Therefore, understanding cellulose dissolution is a very important topic.

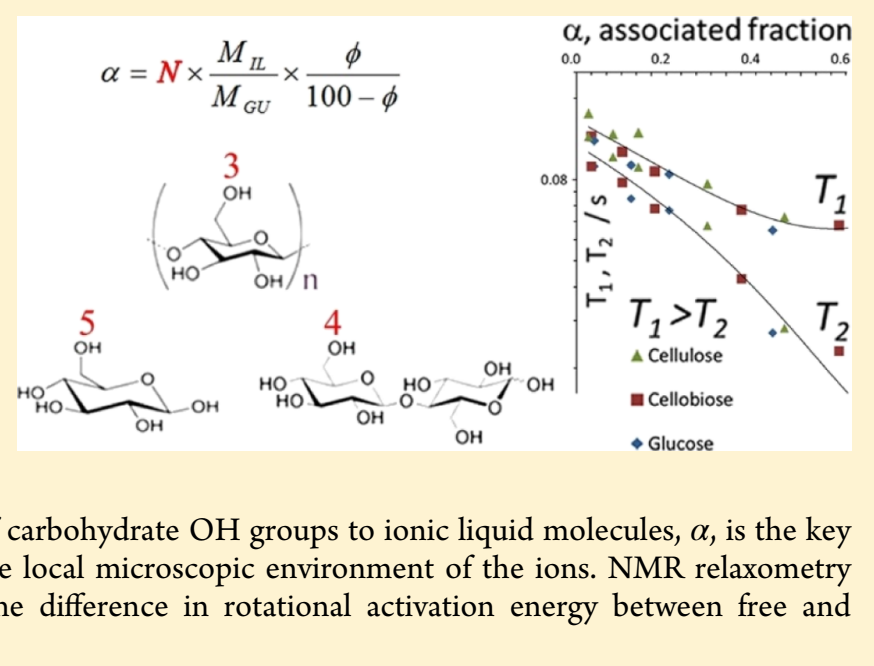

Despite this and the long history of cellulose research, the dissolution of cellulose is still puzzling and consequently generates much research output. ${ }^{12}$ Commonly in the literature, ${ }^{13}$ the reason given for the insolubility of cellulose in water and typical organic solvents is the many intra- and interhydrogen bonds present. ${ }^{14}$ Recently though, the "Lindman hypothesis" reminded the community that cellulose is amphiphilic and that the hydrophobic interactions will also be an important aspect of the solubility of cellulose. ${ }^{15}$

Understanding the dissolution of cellulose is an active topic, involving various experimental tools and molecular modeling. ${ }^{13,16-38}$ For example, Gentile and Olsson ${ }^{17}$ used pulsed field gradient (PFG) nuclear magnetic resonance (NMR) to measure the self-diffusion coefficients in solutions of microcrystalline cellulose and dissolving pulp in aqueous tetrabutylammonium hydroxide (TBAH). It was demonstrated that the TBA + hydrogen ions and the water molecules had a distinct diffusion dependence on the cellulose concentration, indicating quite different molecular interactions with cellulose. One key result was that TBAH binds to cellulose such that there are 1.2 ions associated with each glucose unit, with this number being

Received: July 19, 2018

Revised: August 27, 2018

Published: August 28, 2018 

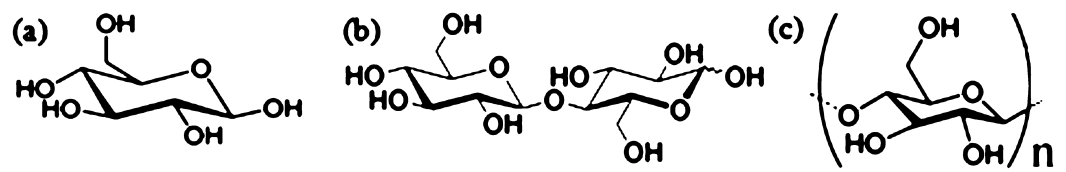

Figure 1. Structure of (a) glucose, (b) cellobiose, and (c) cellulose.

independent of the cellulose molecular weight. An extensive study was carried out by Zhang et al. ${ }^{32}$ in which the solubility of carbohydrates was examined across ILs consisting of 11 different cations and four different anions. The authors used ${ }^{1} \mathrm{H}$ and ${ }^{13} \mathrm{C}$ NMR spectroscopy and tracked the change in chemical shift of the various resonances as a function of the cellobiose concentration. It was shown that the hydrogen bond interaction between the ions and the hydroxyl groups $(\mathrm{OH})$ on the cellobiose is the dominant process in the dissolution. The anions associated with the hydrogen atoms of the $\mathrm{OH}$ groups, whereas the cations associated with the oxygen atoms. Computer simulation work by Bharadwaj et al. ${ }^{16}$ examined glucose and cellobiose in water and three imidazolium-based ILs. It was found that increasing the alkyl chain length of the cation did not alter the solvation of the $\mathrm{OH}$ groups of the cellobiose and glucose by the acetate anion. Fourier transform infrared spectroscopy and multi nuclear NMR spectroscopy were combined with conductivity measurements by Zhang et $\mathrm{al}^{30}$ to determine the molecular interactions in solutions of cellulose and $\mathrm{N}, \mathrm{N}$-dimethylacetamide/ $\mathrm{LiCl}$. They showed that $\mathrm{Li}^{+}-\mathrm{Cl}^{-}$pairs are broken and the $\mathrm{Cl}^{-}$then forms strong hydrogen bonds with the $\mathrm{OH}$ groups of cellulose. Fully atomistic molecular dynamic simulations by Schutt et al. ${ }^{28}$ examined the effect of adding oxygen atoms to the tail of an imidazolium cation on cellulose dissolution, using cellobiose and glucose as model cellulose compounds. The modification of the solvent tail was found to lower its viscosity, with the anion's interactions with the $\mathrm{OH}$ groups of the glucose or cellobiose playing a key role in determining the bulk solution properties. Zhao et al. ${ }^{39}$ used molecular dynamic simulations and quantum chemistry calculations to examine the effects of co-solvent on cellulose dissolution in imidazolium-based ILs; they showed that the dissolution of cellulose is mainly determined by hydrogen bond interactions between the anion and hydroxyl protons of cellulose. From this very brief overview of articles concerning the solubility of cellulose, it is clear that the solvent-cellulose $\mathrm{OH}$ group interactions play a major part in understanding the dissolution of cellulose.

In this work, we will examine solutions of cellulose, cellobiose, and glucose in the IL 1-ethyl-3-methyl-imidazolium acetate $([\mathrm{C} 2 \mathrm{mim}][\mathrm{OAc}])$, currently ${ }^{16}$ one of the most commonly used ILs in cellulose dissolution. Here, we will demonstrate the importance of the molar ratio of carbohydrate hydroxyl groups to ions, showing that this is a key parameter in determining the microscopic dynamics within these systems. The zero shear rate viscosity and low-field $(20 \mathrm{MHz}) \mathrm{NMR}$ relaxometry will be analyzed and then combined through the Stokes-Debye-Einstein relationship. This will enable us to compare and contrast the macroscopic and microscopic properties, showing key differences between the cellulose, cellobiose, and glucose solutions. The NMR relaxometry data will be analyzed using the Bloembergen-Purcell-Pound (BPP) theory, ${ }^{40}$ and it will be argued that the correlation times obtained from this approach correspond to the rotational correlation times of the ions within the solutions. Finally, previously published data $^{37}$ for the ions' self-diffusion coefficients in the very same solutions will be combined with the relaxometry analysis and viscosity results in order to explain the difference in activation energy for diffusional and rotational processes. This analysis will also give information on the additional activation energy for ions to bind to each carbohydrate.

\section{EXPERIMENTAL SECTION}

2.1. Materials and Sample Preparation. Glucose, cellobiose, and cellulose (Avicel PH-101, with a degree of polymerization of 180 as given by the manufacturer) were purchased from Sigma-Aldrich, and prior to dissolution, these materials were dried under vacuum at $70{ }^{\circ} \mathrm{C}$ for a minimum period of $12 \mathrm{~h}$. The structures of glucose, cellobiose, and cellulose $^{14}$ are shown in Figure 1. The IL 1-ethyl-3-methylimidazolium [C2mim][OAc] (97\% purity) was purchased from Sigma-Aldrich and used without any further purification. Neat $[\mathrm{C} 2 \mathrm{mim}][\mathrm{OAc}]$ and three sets of samples (glucose/ cellobiose/cellulose) each with five concentrations of the corresponding carbohydrate $(1,3,5,10$, and 15 wt \%) in [C2mim][OAc] were prepared. Diffusion data from our previous publication ${ }^{37}$ on the same systems are also included in this work.

The sample preparations were made in an MBraun Labmaster 130 atmospheric chamber under nitrogen, providing a dry environment, with the chamber being maintained at a dew point level between -70 and $-40{ }^{\circ} \mathrm{C}$, corresponding to less than $0.5 \mathrm{ppm}$ of water. The $[\mathrm{C} 2 \mathrm{mim}][\mathrm{OAc}]$ and glucose/ cellobiose/cellulose were stirred in a container at $50{ }^{\circ} \mathrm{C}$ for a minimum of $48 \mathrm{~h}$. A small quantity of each carbohydrate $[\mathrm{C} 2 \mathrm{mim}][\mathrm{OAc}]$ solution was then placed in a standard $5 \mathrm{~mm}$ NMR tube within the chamber. Each tube was sealed still within the chamber to prevent moisture contamination and when the samples were not in use they were stored in a desiccator. Karl-Fischer titration indicated that all the samples had less than 0.3 wt \% water. From our previous work, ${ }^{33}$ we found that for water concentrations of $0.5 \mathrm{wt} \%$ and above, a clearly visible water resonance appears in the high-field (Bruker BioSpin $400 \mathrm{MHz}$ ) spectra. All our samples were checked by high-resolution ${ }^{1} \mathrm{H}$ NMR in a Bruker AVANCE II $400 \mathrm{MHz}$ spectrometer for impurities and no degradation or decomposition was observed. ${ }^{41}$

2.2. Low-Field NMR Relaxometry. The spin-lattice relaxation time $T_{1}$ and spin-spin relaxation time $T_{2}$ were determined for each of our samples in steps of $10{ }^{\circ} \mathrm{C}$ between 30 and $70{ }^{\circ} \mathrm{C}$ inclusive, using a $20 \mathrm{MHz}$ Maran benchtop NMR spectrometer. Temperature control was within $\pm 0.1^{\circ} \mathrm{C}$. The inversion recovery method was used to measure $T_{1}$ and the Carr-Purcell-Meiboom-Gill (CPMG) pulse sequence for $T_{2}{ }^{42}$ At each temperature, the samples were left to equilibrate for $10 \mathrm{~min}$ before measurements were recorded. The $90^{\circ}$ pulse width was $3.7 \mu \mathrm{s}$, the signal was averaged across 8 scans, and the repetition time was set to at least $5 \times T_{1}$. In the inversion recovery experiment, a linear increment time step of $\sim 1 / 2 T_{1}$ was used, with 15 increment steps being recorded. For the CPMG sequence, 2000 echoes were used to give a 
total relaxation time of $\sim 5 T_{2}$. Single exponential fits were found to model the NMR relaxation curves very closely for all our results. We estimate the uncertainty on our NMR relaxation times to be less than 5\%.

2.3. Viscosity. A Bohlin Gemini advanced rheometer equipped with a $4^{\circ}-40 \mathrm{~mm}$ cone plate was used to measure the viscosity of the solutions as a function of shear rate. The temperature range was 10 to $100{ }^{\circ} \mathrm{C}$ in $10{ }^{\circ} \mathrm{C}$ increments. A thin film of low viscosity silicon oil was placed around the borders of the measuring cell in order to prevent moisture uptake.

\section{RESULTS AND DISCUSSION}

3.1. Viscosity. In the Supporting Information, Figure S1 shows the viscosity as a function of shear rate for a selection of glucose and cellobiose $-[\mathrm{C} 2 \mathrm{mim}][\mathrm{OAc}]$ solutions at $30{ }^{\circ} \mathrm{C}$, demonstrating a Newtonian flow over a wide range of shear rates. This plateau value was then used as the zero shear rate viscosity, simply "viscosity", in all later analyses. Figure S2 shows that for any given concentration and temperature, cellobiose solution viscosity is very close to that of glucose, well within a $5 \%$ difference. This result was expected as far as both glucose and cellobiose are low-molecular weight compounds and the volumes occupied by each molecule is comparable, at least on the length scales probed by viscosity. We have previously published ${ }^{13}$ the viscosity values for solutions of cellulose-[C2mim][OAc]. Unsurprisingly, because of the polymeric nature of cellulose, its solution viscosity is between one and three orders of magnitudes higher than those of the glucose results presented here; see Figures S3 and S4 in the Supporting Information.

The temperature dependence of the viscosity for the glucose samples is shown in Figure 2, indicating a non-Arrhenius behavior in the large temperature range studied here. Similar nonlinear dependence has already been reported for cellulose-

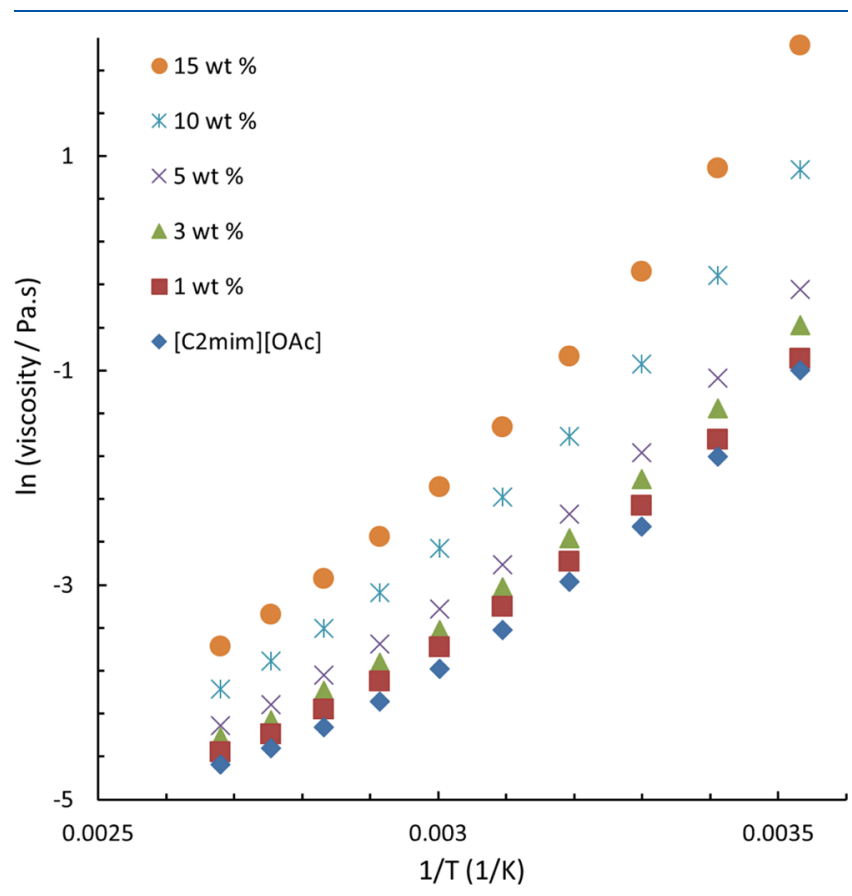

Figure 2. Viscosity of glucose-[C2mim] [OAc] solutions as a function of inverse temperature at different concentrations of glucose. Error bars are within the size of the symbols shown.
$[\mathrm{C} 2 \mathrm{mim}][\mathrm{OAc}]$ and cellulose $-[\mathrm{C} 4 \mathrm{mim}][\mathrm{Cl}]$ solutions, and it was demonstrated that this is induced by the behavior of the IL itself. ${ }^{13}$ For further discussions on "fragile" behavior in liquids, including ILs, the reader is pointed to the seminal work ${ }^{43}$ by Angell.

3.2. NMR Relaxation of lons in Glucose, Cellobiose, and Cellulose Solutions. In Figure $3 \mathrm{a}$ the spin-lattice relaxation times $T_{1}$ are shown for the pure IL [C2mim][OAc], 3 and 15 wt \% carbohydrate weight concentrations. Figure $3 \mathrm{~b}$ shows the spin-spin relaxation times $T_{2}$ for the same samples. As the majority of protons in these samples belong to the IL,
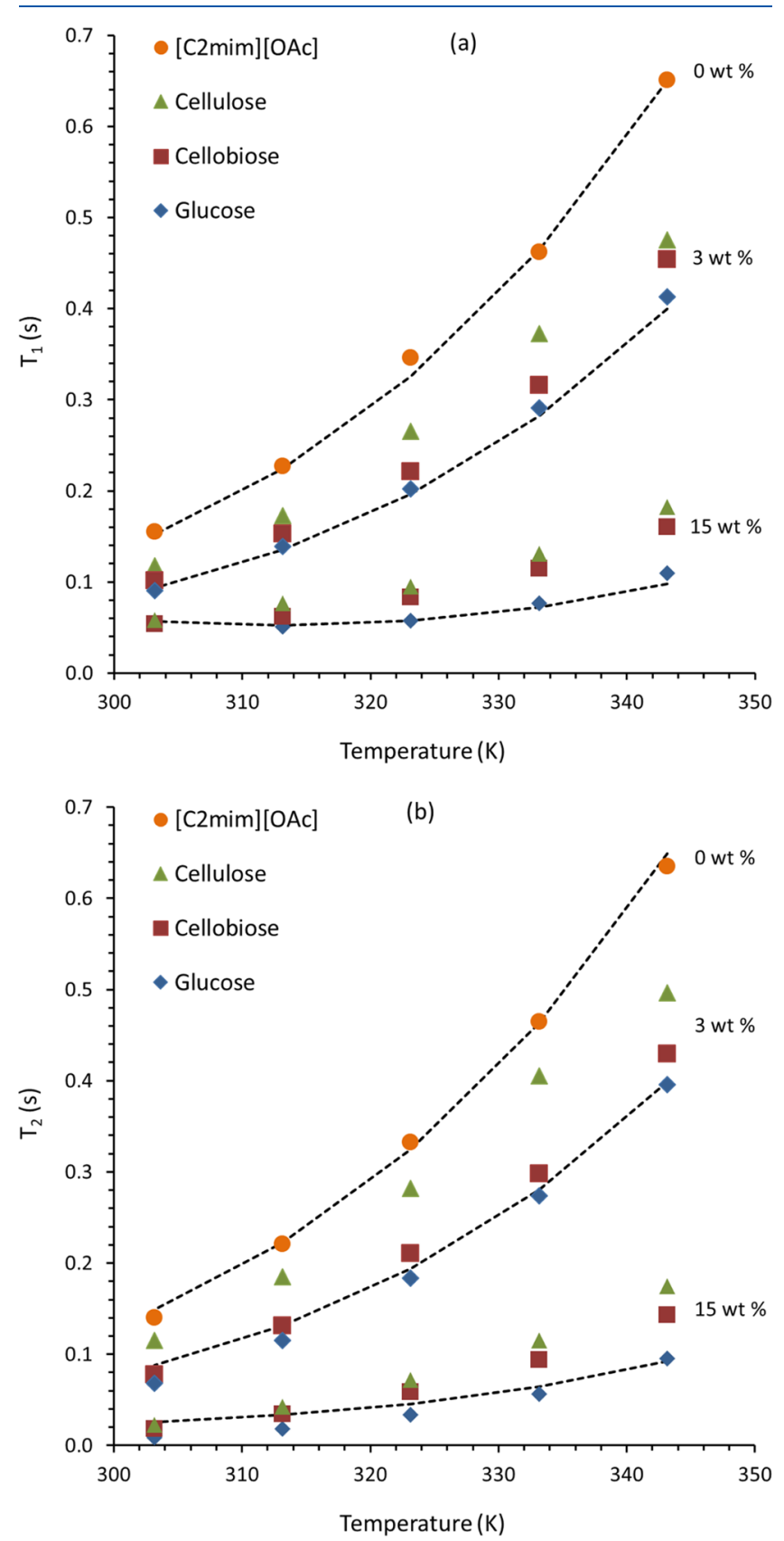

Figure 3. NMR relaxation times (a) $T_{1}$ and (b) $T_{2}$ of [C2mim] [OAc] in glucose, cellobiose, and cellulose solutions as a function of temperature, shown for three weight fractions 0 (pure [C2mim]$[\mathrm{OAc}]), 3$, and $15 \mathrm{wt} \%$. Uncertainties are within the size of the symbols used. The dashed lines are fits of eqs $2 \mathrm{a}$ and $2 \mathrm{~b}$ to the 0,3 , and 15 wt $\%$ glucose data. 
even at the highest carbohydrate concentrations, it will be assumed that the NMR relaxometry is giving information predominantly about the motion of the ions in these solutions. In the $15 \mathrm{wt} \%$ samples, $88 \%$ of the protons belong to the IL molecules. Furthermore, as this is a low-field experiment (20 $\mathrm{MHz}$ ), there is not sufficient chemical resolution to distinguish between cations and anions, and so, the relaxation times are in effect an average across both ions. The various dynamics or mechanisms that contribute to the NMR relaxation will be discussed in more detail later on.

Most of our data have $T_{1}$ approximately equal to $T_{2}$, indicating that the results are within the "liquid" ${ }^{44}$-like NMR response for the majority of our results; only at the highest carbohydrate concentration of 15 wt $\%$ and at the lowest temperature $30{ }^{\circ} \mathrm{C} T_{1}$ is significantly larger than $T_{2}$. This means that with increase in temperature, and corresponding increase in the mobility of the ions, the relaxation times increase. On the other hand, as an increase in the carbohydrate concentration is seen to decrease the NMR relaxation times, this indicates a corresponding decrease in the mobility of the ions. The interactions between the ions and the carbohydrates are therefore reducing the mobility of the ions. It is interesting to note that, weight for weight, at any given temperature, the glucose NMR relaxation times are shorter than the cellobiose times, which again are shorter than the cellulose times. As these NMR relaxation times are related to the mobility of the ions, this result is slightly surprising, as this goes against what would have been expected from the viscosity results. The cellulose samples have the highest viscosity, but according to the NMR relaxometry data, the ions in the cellulose solutions have the highest mobility. Additionally, even though the glucose and cellobiose samples have the same viscosity, weight for weight, they are distinguishable in the NMR experiment, with their NMR relaxation times indicating that the mobility of the ions in these systems is significantly different. These results therefore strongly suggest that the local level or "micro" viscosity experienced by the ions is not simply related to the macroscopically determined zero shear rate viscosity.

In a recent publication, ${ }^{37}$ we measured the self-diffusion coefficients of ions in the very same systems on which we report here. In that previous work, it was found that glucose was the most effective at slowing down the diffusion of the ions and cellulose the least effective, with this perfectly reflecting the change in mobility indicated by the results obtained here on low-field NMR relaxometry. The dependence of $T_{1}$ on the concentration of the solute is displayed in Figure 4, showing glucose to be the most effective and cellulose the least effective at reducing $T_{1}$. Similar results are found for the spin-spin relaxation times and can be found in the Supporting Information; see Figure S5.

In our recent article on diffusion, ${ }^{37}$ we introduced a parameter $\alpha$, termed the "associated fraction" of ions bound to the carbohydrate. Cellulose consists of D-anhydroglucopyranose units (AGU) joined together by $\beta(1 \rightarrow 4)$ glycosidic bonds, each AGU unit within cellulose has three $\mathrm{OH}$ groups (Figure 1). Cellobiose is a disaccharide consisting of two Dglucopyranoses linked by a $\beta(1 \rightarrow 4)$ bond, each D-glucopyranose in cellobiose has four $\mathrm{OH}$ groups. Finally, glucose is a monosaccharide with five $\mathrm{OH}$ groups. The term $\alpha$ corresponds to a molar weight fraction, weighted to the number of $\mathrm{OH}$ groups from the "glucose units" (D-anhydroglucopyranose/Dglucopyranose/D-glucose unit) per [C2mim][OAc] molecule, and is given by $^{37}$

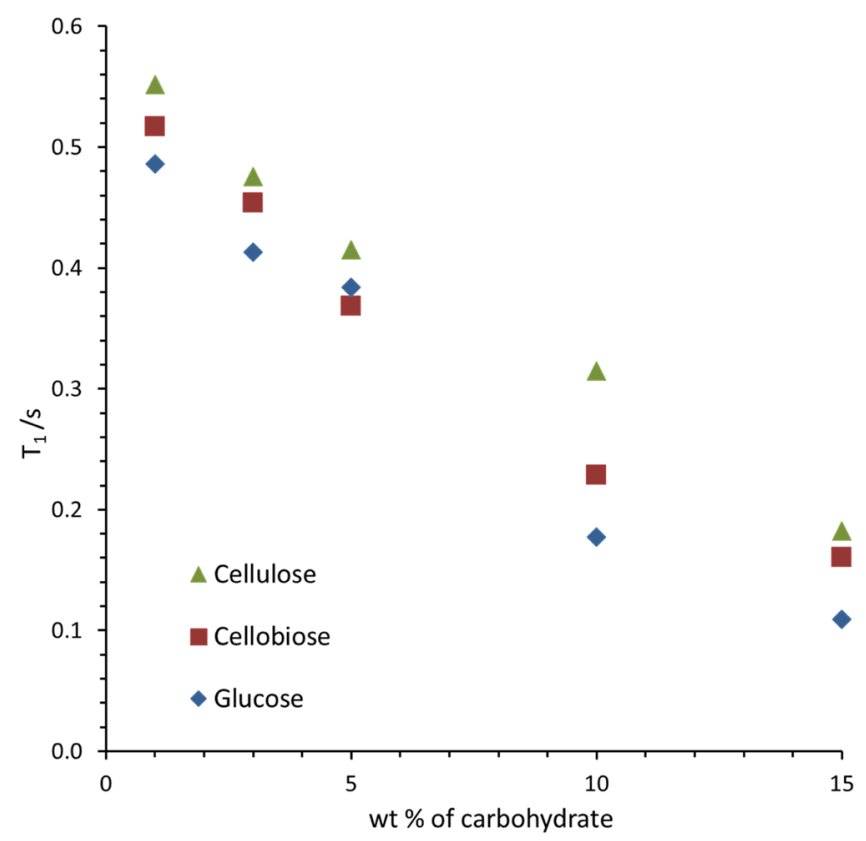

Figure 4. NMR spin-lattice relaxation times $T_{1}$ for glucose, cellobiose, and cellulose as a function of the wt \% of carbohydrate in $[\mathrm{C} 2 \mathrm{mim}][\mathrm{OAc}]$ solutions, at $70{ }^{\circ} \mathrm{C}$. Uncertainties are within the size of the symbols used.

$$
\alpha=N \times \frac{M_{\mathrm{IL}}}{M_{\mathrm{GU}}} \times \frac{\phi}{100-\phi}
$$

where $N$ is the number of $\mathrm{OH}$ groups per "glucose unit" $(5,4$, and 3 for glucose, cellobiose, and cellulose, respectively), $M_{\mathrm{IL}}$ is the molar mass of the IL $(170 \mathrm{~g} / \mathrm{mol}), M_{\mathrm{GU}}$ is the molar mass of a "glucose unit" $(180,171$, and $162 \mathrm{~g} / \mathrm{mol}$ for glucose, cellobiose, and cellulose, respectively), and $\phi$ the weight percent of the carbohydrate in solution. We argued ${ }^{37}$ that the molar ratio $\alpha$ is the fraction of IL molecules involved in dissolving "glucose units" and therefore can be considered as an associated fraction of the IL. When the diffusion data were plotted as a function of $\alpha$, instead of carbohydrate weight fraction, then all the data from the different systems (glucose/ cellobiose/cellulose) fell onto one master curve. In Figure 5, both $T_{1}$ and $T_{2}$ are plotted against $\alpha$ for two temperatures, 30 and $70{ }^{\circ} \mathrm{C}$, showing that master curves are obtained for the NMR relaxation times, as with the published ${ }^{37}$ diffusion data, and this works both in the limit that $T_{1}=T_{2}$ (liquid like regime) and when $T_{1}>T_{2}$ (solid like regime).

NMR relaxation times depend on the dynamics within the system being measured. Rotational and translational motions cause the magnetic fields at the protons to fluctuate. The benchtop analyzer used here operates at a Larmor frequency of $20 \mathrm{MHz}$, making both the $T_{1}$ and $T_{2}$ sensitive to molecular motion and consequent fluctuations at and around this frequency. For ILs, this corresponds to predominantly rotational motion; ${ }^{45}$ for Larmor frequencies above $10 \mathrm{MHz}$, the contribution to the NMR relaxation mechanisms from translational motion becomes less significant as the Larmor frequency is further increased. ${ }^{45-49}$ In this article, we will therefore make the working assumption that rotational motion of the ions is the dominant mechanism for the NMR relaxation and assume a single rotational correlation time $\tau_{\mathrm{R}}$ is responsible for determining both $T_{1}$ and $T_{2}$ for any given temperature and sample. This assumption can later be assessed 

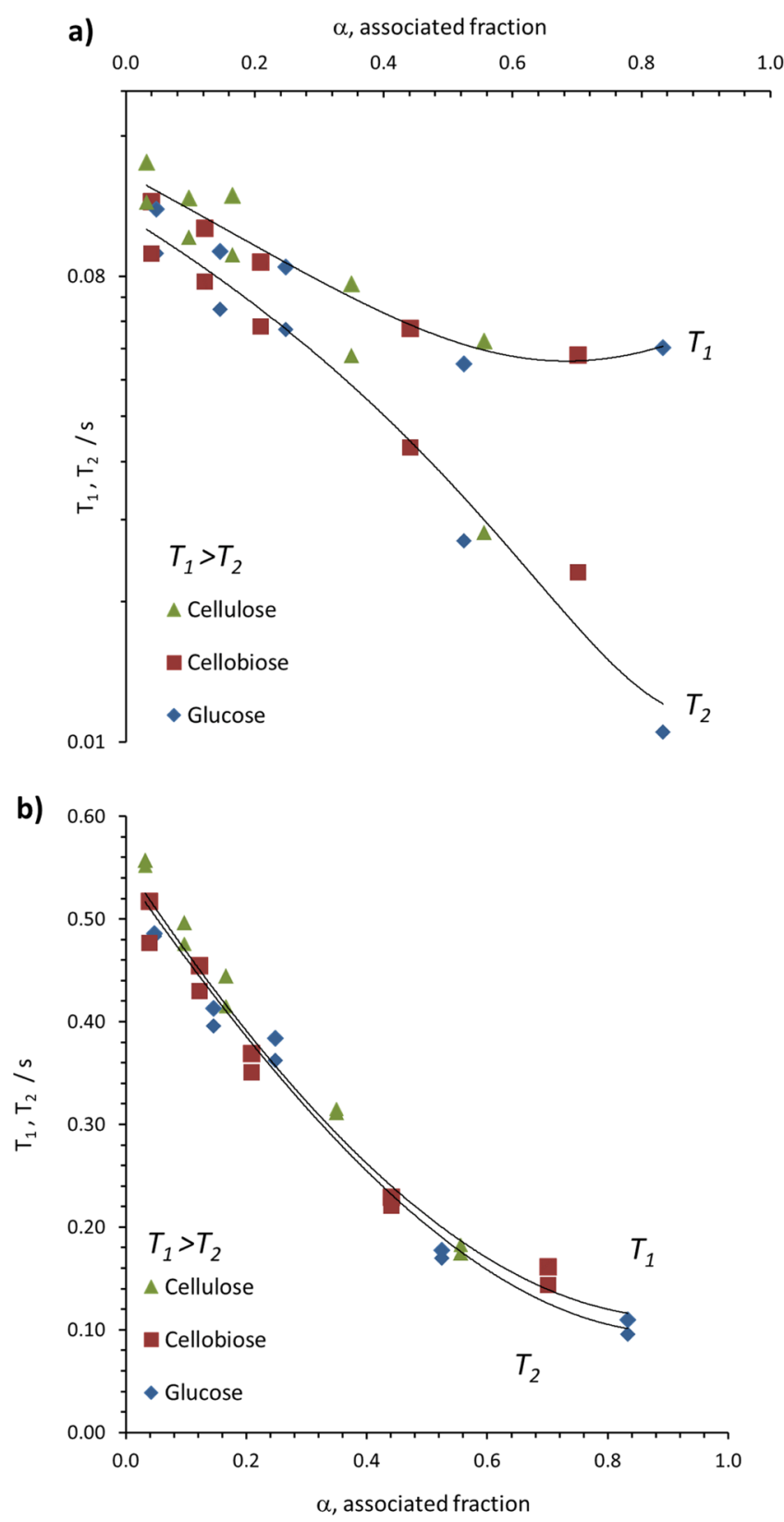

Figure 5. NMR spin-lattice relaxation times $T_{1}$ and $T_{2}$ for [C2mim] [OAc] solutions with glucose, cellobiose, and cellulose as a function of $\alpha$ the associated fraction defined by eq 1 at (a) 30 and (b) $70{ }^{\circ} \mathrm{C}$. Uncertainties are within the size of the symbols used. For all samples and all temperatures, $T_{2}<T_{1}$. Solid lines are given to guide the eye.

by judging how successful it was in: (i) modeling both spinlattice and spin-spin relaxation times simultaneously across all samples and temperatures measured; (ii) following quantitatively the Stokes-Debye-Einstein relationships; and (iii) explaining the difference in activation energies from this analysis and those determined in our prior publication on the ions' self-diffusion coefficients.

According to the BPP approach, the NMR relaxation times can be related to a fluctuation correlation time, here assumed to be a rotational correlation time $\tau_{\mathrm{R}}$ for two protons at fixed distance $r$ apart, as ${ }^{40,50}$

$$
\begin{aligned}
& \frac{1}{T_{1}}=K\left[\frac{\tau_{\mathrm{R}}}{1+\omega_{0}{ }^{2} \tau_{\mathrm{R}}{ }^{2}}+\frac{4 \tau_{\mathrm{R}}}{1+4 \omega_{0}{ }^{2} \tau_{\mathrm{R}}{ }^{2}}\right] \\
& \frac{1}{T_{2}}=\frac{K}{2}\left[3 \tau_{\mathrm{R}}+\frac{5 \tau_{\mathrm{R}}}{1+\omega_{0}{ }^{2} \tau_{\mathrm{R}}{ }^{2}}+\frac{2 \tau_{\mathrm{R}}}{1+4 \omega_{0}{ }^{2} \tau_{\mathrm{R}}{ }^{2}}\right] \\
& K=\frac{3}{10} \gamma^{4} \hbar^{2}\left(\frac{\mu_{0}}{4 \pi}\right)^{2} \frac{1}{r^{6}}
\end{aligned}
$$

where $\omega_{0}$ is the Larmor frequency, $\gamma$ is the gyromagnetic ratio for protons, $\hbar$ is the reduced Planck constant, $\mu_{0}$ is the permeability of free space, and $r$ is a system average or effective distance between protons. For each sample, it will be assumed that there is an activation energy $E_{\mathrm{R}}$ for the rotation correlation time given by

$$
\tau_{\mathrm{R}}=\tau_{0} \exp \left(\frac{E_{\mathrm{R}}}{R T}\right)
$$

where $\tau_{0}$ is a constant sometimes referred to as the high temperature or zero activation energy rotational correlation time, $R$ is the gas constant, and $T$ is the temperature in kelvin. When fitting the data, $\tau_{0}$ and $K$ will be taken as a global fitting parameters as they are related to the moment of inertia of the ions and the distance between protons, respectively, ${ }^{51}$ which should not change significantly with solute type (glucose/ cellobiose/cellulose), concentration, and temperature. For each sample, there will thus be one free parameter, the rotational activation energy $E_{\mathrm{R}}$, and this will have to correctly model both the $T_{1}$ and $T_{2}$ full temperature dependences simultaneously.

In Figure 3, the dashed lines are the resultant fits of eqs $2 \mathrm{a}$ and $2 \mathrm{~b}$ to the pure IL and glucose samples. Fits to all the carbohydrates were equally good as the selection for glucose shown in Figure 3. The global fitting parameters found are $\tau_{0}$ equal to $2.4 \pm 0.1 \times 10^{-15} \mathrm{~s}$ and $K$ equal to $1.7 \pm 0.1 \times 10^{9} \mathrm{~s}^{-2}$. The value of $\tau_{0}$ will be discussed later on. From eq $2 c$, the parameter $K$ gives a very reasonable value for the effective distance between protons $r$ of $2.16 \pm 0.02 \times 10^{-10} \mathrm{~m}$. Comparatively, a rough estimation gives the distance, or lattice spacing, between protons of $2.64 \times 10^{-10} \mathrm{~m}$ (taking the molecular weight of $[\mathrm{C} 2 \mathrm{mim}][\mathrm{OAc}]$ at $170 \mathrm{~g} / \mathrm{mol}$, the density of the IL as $1.1 \mathrm{~g} / \mathrm{cm}^{3},{ }^{33}$ the number of protons per molecule as 14 , and assuming that the protons are on a cubic lattice). This is remarkably close to the value determined through the NMR relaxometry analysis, especially given such a simple calculation, and thus supports the quantitative validity of the BPP analysis applied here.

In our recent article, ${ }^{37}$ we argued that the ions in these carbohydrate systems behaved as an "ideal mixture" of free and associated ions. It was shown theoretically that for this ideal mixture rule to correctly describe the diffusion of the ions, the activation energy for their translational diffusion needed to be linear with respect to the associated fraction $\alpha$. This was verified experimentally for diffusion of $[\mathrm{C} 2 \mathrm{mim}][\mathrm{OAc}]$ in glucose/cellobiose/cellulose solutions. In Figure 6, we likewise plot the rotational activation energy as determined from the BPP analysis of the low-field relaxometry data. The activation energy results all follow a linear dependence as a function of associated fraction of ions, suggesting that the rotational motion also obeys an ideal mixture rule. Because both rotational and translational motions are governed by $\alpha$, the effective local microscopic viscosity experienced by the ions 


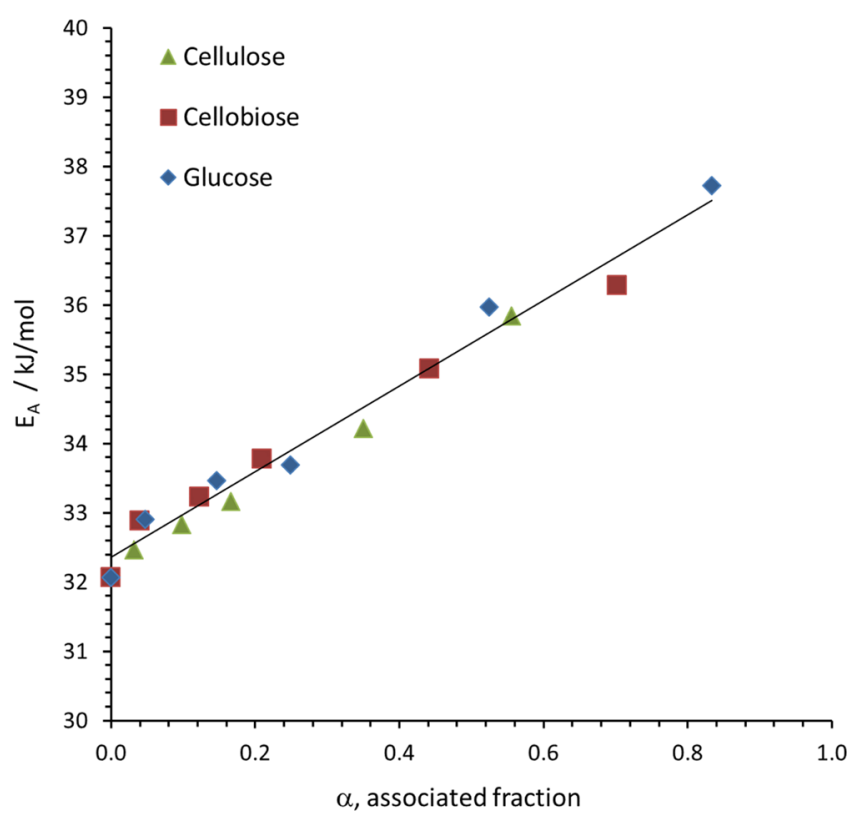

Figure 6. Activation energies of the correlation time $\tau$, found from BPP analysis using eqs $2 \mathrm{a}, 2 \mathrm{~b}$ and 3 , plotted against associated fraction. The straight line is a fit to all the data presented, with an $R^{2}$ of 0.98. Error bars are within the size of the symbols shown. The global fitting parameter is $\tau_{0}=2.4 \pm 0.1 \times 10^{-15} \mathrm{~s}$, which gives $\tau_{\mathrm{R}}$ values $\sim 0.1 \mathrm{~ns}$ across the temperature range studied here.

must also be determined by this parameter. In other words, the number of carbohydrate $\mathrm{OH}$ groups within these solutions dictates the dynamics of the ions.

The extra "cost" in terms of additional activation energy for rotation of an ion due to its association with an $\mathrm{OH}$ group from a carbohydrate molecule is given directly by the gradient of the solid line in Figure 6 as $6.2 \pm 0.5 \mathrm{~kJ} / \mathrm{mol}$, which is reasonably close to the values found previously ${ }^{37}$ for the increase in diffusional activation energy for being associated with an $\mathrm{OH}$ group of a carbohydrate molecule of $8.2 \pm 0.4$ and $7.6 \pm 0.4 \mathrm{~kJ} / \mathrm{mol}$ for the anion and cation, respectively. Therefore, there is across the full range of associated fractions $\alpha$ an approximately constant difference between the rotational activation energy and the corresponding diffusional activation energy of $14 \pm 2 \mathrm{~kJ} / \mathrm{mol}^{37}$ with the diffusional motion having the higher energy barrier.

In the seminal work by Powell, Roseveare, and Eyring, a theory of viscosity, diffusion, thermal, and ionic conductivities in terms of a statistical mechanical theory for the reaction rate was developed. ${ }^{52}$ For flow to take place, a single molecule moves past its neighbor and falls into a vacant equilibrium position, termed a hole or vacancy. An activation energy is required for a molecule to jump over its neighbor. The authors showed that there was a close link between viscous flow and vaporization because the same bonds that need to be broken for flow to take place are required to be broken for vaporization. ${ }^{52}$ In mixtures, the ease of molecular flow is not determined predominantly by its own properties, but by the "solvent" or surrounding molecules that must contribute holes for it to flow into. In 1968, O’Reilly investigated the diffusion coefficients and rotational correlation times of several polar liquids. ${ }^{53}$ In his work, he argued that the difference between the activation energy for rotational motion and that for diffusional motion was due to the additional cost of creating the vacancy (or hole) for the diffusing molecule to move into.
For both rotational and diffusional motion to occur, all the close neighboring bonds must be broken, but for the diffusional translation, there is the extra cost of creating the hole. This can be written mathematically as, ${ }^{53}$

$$
D=D_{0} \exp \left(-\frac{E_{\mathrm{R}}+E_{\text {hole }}}{R T}\right)
$$

where $E_{\text {hole }}$ is the additional activation energy needed to create a vacancy into which the diffusing molecule can move into and, as argued above, has a value of $14 \pm 2 \mathrm{~kJ} / \mathrm{mol}$.

If we now continue to assume that the fluctuation correlation times $\tau_{\mathrm{R}}$ found from the BPP analysis are due to the rotational motion of the ions, it is then possible to theoretically predict the value for $E_{\text {hole }}$ through the StokesDebye-Einstein relationship ${ }^{51,54}$

$$
\tau_{\mathrm{R}}=\frac{4 \pi R_{\mathrm{H}}{ }^{3} \eta}{3 k T}
$$

where $R_{\mathrm{H}}$ is the effective hydrodynamic radius of the molecule and $k$ is the Boltzmann constant.

The ratio of the viscosity to the temperature in eq 5 can be eliminated in favor of the diffusion coefficient through the Stokes-Einstein formula ${ }^{51,54}$

$$
D=\frac{k T}{6 \pi R_{\mathrm{H}} \eta}
$$

This gives

$$
\tau=\frac{2 R_{\mathrm{H}}^{2}}{9 D}
$$

Then, combining this result with eqs 3 and 4 obtains

$$
E_{\text {hole }}=R T \ln \left(\frac{9}{2} \frac{D_{0} \tau_{0}}{R_{\mathrm{H}}^{2}}\right)
$$

In the BPP analysis, we obtain only one correlation time $\tau_{\mathrm{R}}$ and this is an effective average rotational time scale for the cations and anions in our system. To use eq 8 to estimate $E_{\text {hole }}$, we take: (i) the average value of $D_{0}$ of $1.5 \times 10^{-3} \mathrm{~m}^{2} \mathrm{~s}^{-1}$ for the cation and anion $\left(1.4 \pm 0.2 \times 10^{-3}\right.$ and $1.6 \pm 0.2 \times 10^{-3} \mathrm{~m}^{2}$ $\mathrm{s}^{-1}$, respectively; ${ }^{37}$ ) and (ii) the average value of hydrodynamic radii $R_{\mathrm{H}}, 2.5 \times 10^{-10} \mathrm{~m}$, of the cation and anion $\left(2.8 \times 10^{-10}\right.$ and $2.2 \times 10^{-10} \mathrm{~m}$, respectively $\left.{ }^{37}\right)$ and $\tau_{0}=2.4 \pm 0.1 \times 10^{-15} \mathrm{~s}$ from the abovementioned BPP analysis. Because our measurements span from 30 to $70{ }^{\circ} \mathrm{C}$, we set $T$ in eq 8 to an average value of $320 \mathrm{~K}\left(50{ }^{\circ} \mathrm{C}\right)$; all the temperatures in this study are within $6 \%$ of this middle value. Finally, combining all these parameters into eq 8 gives a prediction for $E_{\text {hole }}$ of $15 \mathrm{~kJ} / \mathrm{mol}$, which is remarkably close to the measured value of $14 \pm 2 \mathrm{~kJ} /$ mol. This is strong support for taking the correlations time found from the relaxometry measurements through the BPP analysis as rotational correlation times; it also indicates that the parameters found from this approach are quantitatively correct.

When $\tau_{\mathrm{R}}$ and $D$ are compared, as in the abovementioned analysis, then one microscopic term is being compared with another microscopic term. The quantitative agreement found above indicates that there is one effective microscopic viscosity that determines both the rotational and translational motion of the ions. This local microscopic viscosity can be altered by either varying the temperature and/or changing the number of solute $\mathrm{OH}$ groups for the ions to interact with, and it makes no difference whether those $\mathrm{OH}$ groups come from glucose, or 
cellobiose or indeed cellulose molecules. However, what is interesting now is to compare the microscopic environment with the macroscopic one. This can be done by plotting $T_{1}$, which as we have shown, is determined by microscopic rotational motion, against $T / \eta$, as is done in Figure 7 .

The zero shear rate viscosity $\eta$ is a macroscopic term, measured using a rheometer, and thus here, large scale effects relative to the size of ions, such as polymer entanglements, play a significant role in determining the resultant macroscopic viscosity. However, they play an almost insignificant role in determining the microscopic viscosity that determines the rotational and translational motions of the ions: for glucose and cellobiose, all data at various concentrations fall on one master plot (Figure $7 \mathrm{a}, \mathrm{b}$, respectively), which is not the case for cellulose (Figure 7c). For the glucose and cellobiose solutions, the macroscopic and microscopic viscosities are proportional to each other, both being affected in a similar manner by changes in temperature and solute concentration. This is not the case for the cellulose solutions, with macroscopic viscosity dramatically increasing when macromolecules $^{55}$ are added into a solvent, especially above the overlap concentration (here, around ${ }^{56} 1$ wt \%), as expected. On the length scale of the ions, the local microviscosity within the cellulose samples is similar to that of the glucose and cellobiose samples; the key determining factor on these local length scales is the density of $\mathrm{OH}$ groups that the ions interact with.

\section{CONCLUSIONS}

In this work, we analyzed carbohydrate solutions in IL [C2mim] $[\mathrm{OAc}]$ by measuring: (i) the relaxation times of protons of IL probed by NMR relaxometry at $20 \mathrm{MHz}$ and (ii) viscosity of these solutions. The carbohydrates were glucose, cellobiose, and cellulose, and each set of solutions was of five concentrations $(1,3,5,10$, and 15 wt \%). Each solution was measured at temperatures from 30 to $70{ }^{\circ} \mathrm{C}$. Cellulose is found to be the most effective in increasing the solution viscosity as compared to glucose and cellobiose. In contrast, glucose was found to be the most effective in reducing the NMR relaxation times and cellulose the least effective. As the NMR relaxation times can be related to the mobility of the ions, this indicates that the ions in the most viscous set of samples have, counterintuitively, the highest mobility. A similar surprising result was found when these samples were investigated previously $^{37}$ using PFG NMR to determine the self-diffusion coefficients of the ions.

We demonstrated that it is the number of carbohydrate $\mathrm{OH}$ groups per repeating "glucose" unit that determines the mobility of the ions. We introduced ${ }^{37}$ the parameter $\alpha$, which quantifies the molar ratio of $\mathrm{OH}$ groups per IL molecule. As glucose has more $\mathrm{OH}$ groups per repeat unit, then for any corresponding weight concentrations, these samples will have a higher number of $\mathrm{OH}$ groups for the ions to interact with. It is these interactions that slow down the rotational and translational motion of the ions and, as a consequence, this loss of mobility reduces the NMR relaxation times. When the NMR relaxation times are plotted not as a function of weight concentration, but instead against $\alpha$, then all data fall on master curves independent of particular carbohydrate. This is strong evidence that the molar density of $\mathrm{OH}$ groups is the most important factor in determining the microscopic environment of the ions.
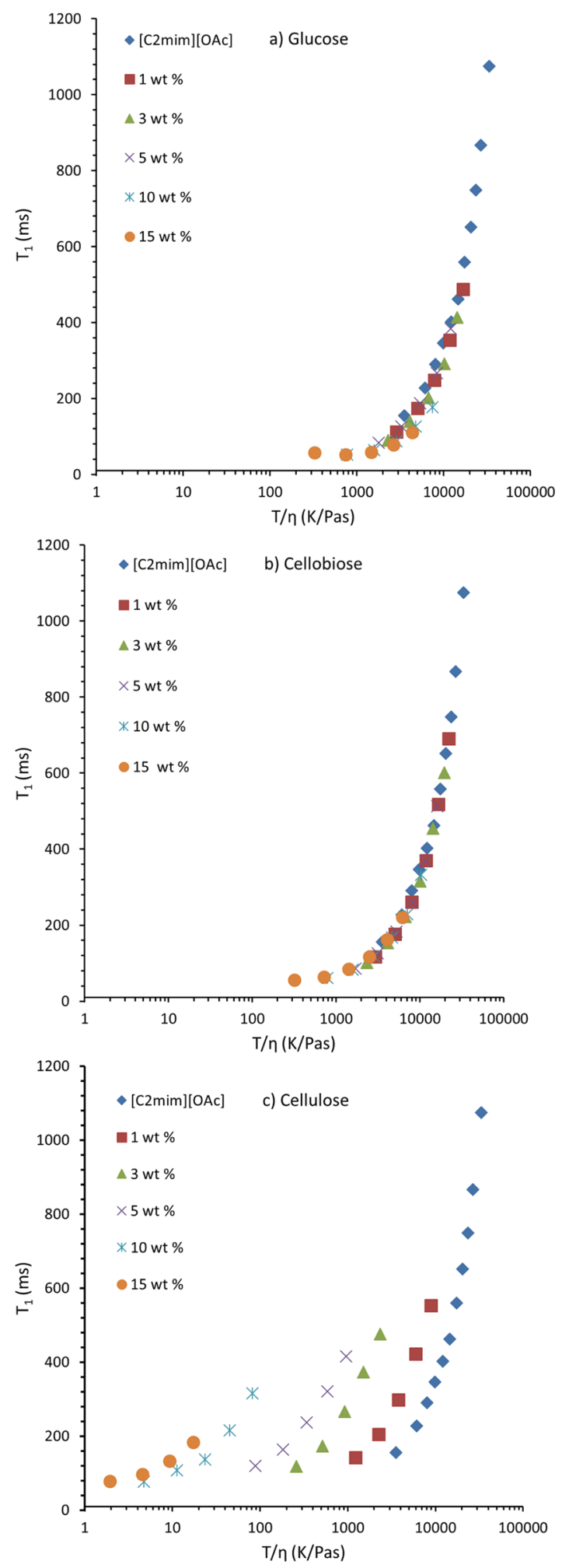

Figure 7. Spin-lattice relaxation time $T_{1}$ against temperature over viscosity for (a) glucose, (b) cellobiose, and (c) cellulose samples at various carbohydrate concentrations in wt \%. Error bars are within the size of the symbols shown.

The NMR relaxation times were analyzed in terms of the theoretical $^{40}$ work of BPP. For each sample at each temperature, a correlation time $\tau_{\mathrm{R}}$ was found. The activation energies for this correlation time were shown to be linearly dependent on $\alpha$, and this reveals that these solutions can be 
considered as ideal mixtures of associated and nonassociated ions.

For an associated ion, there is the additional cost for rotation, $6.2 \pm 0.5 \mathrm{~kJ} / \mathrm{mol}$, compared to that for a free ion. From previous work on the diffusion of ions in these same systems, ${ }^{37}$ translational motion involves a higher activation energy, an extra $14 \pm 2 \mathrm{~kJ} / \mathrm{mol}$, than that for rotational motion obtained in this work. We interpreted this additional barrier stemming from the need of a hole or vacancy to form in translational motion. ${ }^{52}$ By using Stokes-Debye-Einstein equations linking viscosity to rotation and diffusion, combined with the fitting parameters from the NMR relaxometry analysis, it was possible to predict this additional cost quite accurately $(15 \mathrm{~kJ} / \mathrm{mol})$. This supports our interpretation that the correlation times $\tau_{\mathrm{R}}$ found are predominantly arising from rotational motion. The success of eq 8 is quite remarkable, in that it uses two parameters $D_{0}$ and $\tau_{0}$ that are not usually the subjects of Arrhenius-type analysis, which when coupled with the average hydrodynamic radius of the ions calculates the activation energy in forming a vacancy for translational motion. This then predicts the difference between how diffusion and rotation change as a function of temperature. This is strong evidence for the quantitative nature of our analysis and supports our interpretation that the NMR relaxometry can be related to the rotational motion of the ions.

Finally, this work highlights important differences between what is occurring microscopically and macroscopically in a carbohydrate IL solution. Macroscopically, the viscosity depends on the volume occupied by the solute. Microscopically, the dominant factor is the number of $\mathrm{OH}$ groups on a carbohydrate molecule, the effect of which can be quantified by the associated fraction $\alpha$.

\section{ASSOCIATED CONTENT}

\section{S Supporting Information}

The Supporting Information is available free of charge on the ACS Publications website at DOI: 10.1021/acs.jpcb.8b06939.

Viscosity against the shear rate of carbohydrate[C2mim] [OAc] solutions; viscosity at 10,60 , and 100 ${ }^{\circ} \mathrm{C}$ for the glucose and cellobiose in $[\mathrm{C} 2 \mathrm{mim}][\mathrm{OAc}]$ solutions as a function of carbohydrate concentration; viscosity of cellulose $[\mathrm{C} 2 \mathrm{mim}][\mathrm{OAc}]$ solutions against the cellulose concentration; viscosity ratio of cellulose to glucose solutions in $[\mathrm{C} 2 \mathrm{mim}][\mathrm{OAc}]$ against carbohydrate concentration; and NMR spin-spin relaxation times $T_{2}$ for glucose, cellobiose, and cellulose as a function of the wt \% of carbohydrate in [C2mim] [OAc] solutions at $70{ }^{\circ} \mathrm{C}(\mathrm{PDF})$

\section{AUTHOR INFORMATION}

\section{Corresponding Author}

*E-mail: m.e.ries@leeds.ac.uk.

\section{ORCID}

Michael E. Ries: 0000-0002-8050-3200

Stephen M. Green: 0000-0002-1249-6950

Tatiana Budtova: 0000-0003-1835-2146

\section{Notes}

The authors declare no competing financial interest.

\section{ACKNOWLEDGMENTS}

S.M.G. is funded by an EPSRC CASE Award (EP/I501495/1) with Innovia Films (Wigton, Cumbria, CA7 9BG, UK). M.E.R. is a Royal Society Industry Fellow (IF120090). The data for this article can be found at doi: https://doi.org/10.5518/369.

\section{REFERENCES}

(1) Walden, P. Molecular magnitude and electrical conductivity of some fused salts. Bulletin de l'Academie Imperiale des Sciences de St. Petersbourg; Commissionnaires de l'Académie impériale des sciences, 1914, 405-422.

(2) Angell, C. A.; Ansari, Y.; Zhao, Z. F. Ionic liquids: past, present and future. Faraday Discuss. 2012, 154, 9-27.

(3) Plechkova, N. V.; Seddon, K. R. Applications of ionic liquids in the chemical industry. Chem. Soc. Rev. 2008, 37, 123-150.

(4) Short, P. L. Out of the Ivory tower. Chem. Eng. News 2006, 84, $15-21$.

(5) Wang, H.; Gurau, G.; Rogers, R. D. Ionic liquid processing of cellulose. Chem. Soc. Rev. 2012, 41, 1519-1537.

(6) Welton, T. Room-temperature ionic liquids. Solvents for synthesis and catalysis. Chem. Rev. 1999, 99, 2071-2083.

(7) Zhu, S.; Chen, R.; Wu, Y.; Chen, Q.; Zhang, X.; Yu, Z. A minireview on greenness of ionic liquids. Chem. Biochem. Eng. Q. 2009, 23, 207-211 see https://hrcak.srce.hr/38322.

(8) Zhu, S.; Wu, Y.; Chen, Q.; Yu, Z.; Wang, C.; Jin, S.; Ding, Y.; $\mathrm{Wu}, \mathrm{G}$. Dissolution of cellulose with ionic liquids and its application: a mini-review. Green Chem. 2006, 8, 325-327.

(9) Graenacher, C. Cellulose solution. US Patent 1,943,176, 1934.

(10) Swatloski, R. P.; Spear, S. K.; Holbrey, J. D.; Rogers, R. D. Dissolution of cellose with ionic liquids. J. Am. Chem. Soc. 2002, 124, 4974-4975.

(11) Klemm, D.; Heublein, B.; Fink, H.-P.; Bohn, A. Cellulose: Fascinating biopolymer and sustainable raw material. Angew. Chem., Int. Ed. 2005, 44, 3358-3393.

(12) Medronho, B.; Romano, A.; Miguel, M. G.; Stigsson, L.; Lindman, B. Rationalizing cellulose (in)solubility: reviewing basic physicochemical aspects and role of hydrophobic interactions. Cellulose 2012, 19, 581-587.

(13) Sescousse, R.; Le, K. A.; Ries, M. E.; Budtova, T. Viscosity of cellulose-imidazolium-based ionic liquid solutions. J. Phys. Chem. B 2010, 114, 7222-7228.

(14) French, A. D. Glucose, not cellobiose, is the repeating unit of cellulose and why that is important. Cellulose 2017, 24, 4605-4609.

(15) Glasser, W. G.; Atalla, R. H.; Blackwell, J.; Brown, R. M.; Burchard, W.; French, A. D.; Klemm, D. O.; Nishiyama, Y. About the structure of cellulose: debating the Lindman hypothesis. Cellulose 2012, 19, 589-598.

(16) Bharadwaj, V. S.; Schutt, T. C.; Ashurst, T. C.; Maupin, C. M. Elucidating the conformational energetics of glucose and cellobiose in ionic liquids. Phys. Chem. Chem. Phys. 2015, 17, 10668-10678.

(17) Gentile, L.; Olsson, U. Cellulose-solvent interactions from selfdiffusion NMR. Cellulose 2016, 23, 2753-2758.

(18) Green, S. M.; Ries, M. E.; Moffat, J.; Budtova, T. NMR and rheological study of anion size influence on the properties of two imidazoliumbased ionic liquids. Sci. Rep. 2017, 7, 8968.

(19) Han, N.; Wang, X. F.; Qu, T. S.; Qian, Y. Q.; Lu, Y. H. Preparation and properties of cellulose benzoate and preliminary exploration about cellulose benzoate-g-polyoxyethylene(2) hexadecyl ether. Chem. J. Chin. Univ. 2017, 38, 1099-1106.

(20) Hedlund, A.; Köhnke, T.; Theliander, H. Diffusion in ionic liquid-cellulose solutions during coagulation in water: mass transport and coagulation rate measurements. Macromolecules 2017, 50, 87078719.

(21) Hegde, G. A.; Bharadwaj, V. S.; Kinsinger, C. L.; Schutt, T. C.; Pisierra, N. R.; Maupin, C. M. Impact of water dilution and cation tail length on ionic liquid characteristics: Interplay between polar and non-polar interactions. J. Chem. Phys. 2016, 145, 064504. 
(22) Isik, M.; Sardon, H.; Mecerreyes, D. Ionic liquids and cellulose: dissolution, chemical modification and preparation of new cellulosic materials. Int. J. Mol. Sci. 2014, 15, 11922-11940.

(23) Jia, L.; Pedersen, C. M.; Qiao, Y.; Deng, T.; Zuo, P.; Ge, W.; Qin, Z.; Hou, X.; Wang, Y. Glucosamine condensation catalyzed by 1ethyl-3-methylimidazolium acetate: mechanistic insight from NMR spectroscopy. Phys. Chem. Chem. Phys. 2015, 17, 23173-23182.

(24) Kimura, M.; Shinohara, Y.; Takizawa, J.; Ren, S.; Sagisaka, K.; Lin, Y.; Hattori, Y.; Hinestroza, J. P. Versatile molding process for tough cellulose hydrogel materials. Sci. Rep. 2015, 5, 16266.

(25) Norman, S. E.; Turner, A. H.; Holbrey, J. D.; Youngs, T. G. A. Solvation structure of uracil in ionic liquids. ChemPhysChem 2016, 17, 3923-3931.

(26) Radhi, A.; Le, K. A.; Ries, M. E.; Budtova, T. Macroscopic and Microscopic Study of 1-Ethyl-3-methyl-imidazolium Acetate-DMSO Mixtures. J. Phys. Chem. B 2015, 119, 1633-1640.

(27) Schuermann, J.; Huber, T.; LeCorre, D.; Mortha, G.; Sellier, M.; Duchemin, B.; Staiger, M. P. Surface tension of concentrated cellulose solutions in 1-ethyl-3-methylimidazolium acetate. Cellulose 2016, 23, 1043-1050.

(28) Schutt, T. C.; Bharadwaj, V. S.; Hegde, G. A.; Johns, A. J.; Maupin, C. M. In silico insights into the solvation characteristics of the ionic liquid 1-methyltriethoxy-3-ethylimidazolium acetate for cellulosic biomass. Phys. Chem. Chem. Phys. 2016, 18, 23715-23726.

(29) Singh, V.; Panda, S.; Kaur, H.; Banipal, P. K.; Gardas, R. L.; Banipal, T. S. Solvation behavior of monosaccharides in aqueous protic ionic liquid solutions: Volumetric, calorimetric and NMR spectroscopic studies. Fluid Phase Equilib. 2016, 421, 24-32.

(30) Zhang, C.; Liu, R.; Xiang, J.; Kang, H.; Liu, Z.; Huang, Y. Dissolution mechanism of cellulose in $\mathrm{N}, \mathrm{N}$-dimethylacetamide/ lithium chloride: revisiting through molecular interactions. J. Phys. Chem. B 2014, 118, 9507-9514.

(31) Zhang, J.; Wu, J.; Yu, J.; Zhang, X.; He, J.; Zhang, J. Application of ionic liquids for dissolving cellulose and fabricating cellulose-based materials: state of the art and future trends. Mater. Chem. Front. 2017, 1, 1273-1290.

(32) Zhang, J.; Xu, L.; Yu, J.; Wu, J.; Zhang, X.; He, J.; Zhang, J. Understanding cellulose dissolution: effect of the cation and anion structure of ionic liquids on the solubility of cellulose. Sci. China: Chem. 2016, 59, 1421-1429.

(33) Hall, C. A.; Le, K. A.; Rudaz, C.; Radhi, A.; Lovell, C. S.; Damion, R. A.; Budtova, T.; Ries, M. E. Macroscopic and microscopic study of 1-ethyl-3-methyl-imidazolium acetate-water mixtures. J. Phys. Chem. B 2012, 116, 12810-12818.

(34) Lovell, C. S.; Walker, A.; Damion, R. A.; Radhi, A.; Tanner, S. F.; Budtova, T.; Ries, M. E. Influence of cellulose on ion diffusivity in 1-ethyl-3-methyl-imidazolium acetate cellulose solutions. Biomacromolecules 2010, 11, 2927-2935.

(35) Ries, M. E.; Budtova, T.; Radhi, A. NMR relaxometry, diffusion, and rheology studies of carbohydrates in ionic liquids. Abstracts of Papers of the American Chemical Society, 2014, Vol. 247, 258-CELL.

(36) Ries, M. E.; Budtova, T.; Radhi, A.; Ovenden, J.; Keating, A.; Parker, O. NMR studies of carbohydrate solvation in the room temperature ionic liquid 1-ethyl-3-methyl-imidazolium acetate. Abstracts of Papers of the American Chemical Society, 2013, Vol. 245, 531-POLY.

(37) Ries, M. E.; Radhi, A.; Keating, A. S.; Parker, O.; Budtova, T. Diffusion of 1-ethyl-3-methyl-imidazolium acetate in glucose, cellobiose, and cellulose solutions. Biomacromolecules 2014, 15, 609-617.

(38) D’Agostino, C.; Mantle, M. D.; Mullan, C. L.; Hardacre, C.; Gladden, L. F. Diffusion, ion pairing and aggregation in 1-ethyl-3methylimidazolium-based ionic liquids studied by H1 and F19 PFG NMR: effect of temperature, anion and glucose dissolution. ChemPhysChem 2018, 19, 1081-1088.

(39) Zhao, Y.; Liu, X.; Wang, J.; Zhang, S. Insight into the cosolvent effect of cellulose dissolution in imidazolium-based ionic liquid systems. J. Phys. Chem. B 2013, 117, 9042-9049.
(40) Bloembergen, N.; Purcell, E. M.; Pound, R. V. Relaxation effects In nuclear magnetic resonance absorption. Phys. Rev. 1948, 73, $679-712$.

(41) Clough, M. T.; Geyer, K.; Hunt, P. A.; Son, S.; Vagt, U.; Welton, T. Ionic liquids: not always innocent solvents for cellulose. Green Chem. 2015, 17, 231-243.

(42) Carr, H. Y.; Purcell, E. M. Effects of diffusion on free precession in nuclear magnetic resonance experiments. Phys. Rev. 1954, 94, 630638.

(43) Angell, C. A. Formation of glasses from liquids and biopolymers. Science 1995, 267, 1924-1935.

(44) Callaghan, P. T. Principles of Nuclear Magnetic Resonance Microscopy; Clarendon Press: Oxford, 1992; p 492.

(45) Daniel, C. I.; Chávez, F. V.; Feio, G.; Portugal, C. A. M.; Crespo, J. G.; Sebastião, P. J. H1 NMR relaxometry, viscometry, and PFG NMR studies of magnetic and nonmagnetic ionic liquids. J. Phys. Chem. B 2013, 117, 11877-11884.

(46) Daniel, C. I.; Chávez, F. V.; Portugal, C. A. M.; Crespo, J. G.; Sebastião, P. J. H1 NMR relaxation study of a magnetic ionic liquid as a potential contrast agent. J. Phys. Chem. B 2015, 119, 11740-11747.

(47) Driver, G. W.; Huang, Y.; Laaksonen, A.; Sparrman, T.; Wang, Y.-L.; Westlund, P.-O. Correlated/non-correlated ion dynamics of charge-neutral ion couples: the origin of ionicity in ionic liquids. Phys. Chem. Chem. Phys. 2017, 19, 4975-4988.

(48) Neves, L. A.; Sebastião, P. J.; Coelhoso, I. M.; Crespo, J. G. Proton NMR relaxometry study of nafion membranes modified with ionic liquid cations. J. Phys. Chem. B 2011, 115, 8713-8723.

(49) Seyedlar, A. O.; Stapf, S.; Mattea, C. Dynamics of the ionic liquid 1-butyl-3-methyl-imidazolium bis(trifluoromethylsulphonyl)imide studied by nuclear magnetic resonance dispersion and diffusion. Phys. Chem. Chem. Phys. 2015, 17, 1653-1659.

(50) Blicharska, B.; Peemoeller, H.; Witek, M. Hydration water dynamics in biopolymers from NMR relaxation in the rotating frame. J. Magn. Reson. 2010, 207, 287-293.

(51) Carper, W. R.; Mains, G. J.; Piersma, B. J.; Mansfield, S. L.; Larive, C. K. C-13 NMR relaxation and H1 diffusion (DOSY) studies of an acidic chloroaluminate melt. J. Phys. Chem. 1996, 100, 47244728.

(52) Powell, R. E.; Roseveare, W. E.; Eyring, H. Diffusion, thermal conductivity, and viscous flow of liquids. Ind. Eng. Chem. 1941, 33, 430-435.

(53) O'Reilly, D. E. Self-diffusion coefficients and rotational correlation times in polar liquids. J. Chem. Phys. 1968, 49, 5416-5420.

(54) Antony, J. H.; Dölle, A.; Mertens, D.; Wasserscheid, P.; Carper, W. R.; Wahlbeck, P. G. C13 NMR relaxation rates in the ionic liquid 1-methyl-3-nonylimidazolium hexafluorophosphate. J. Phys. Chem. A 2005, 109, 6676-6682.

(55) Staudinger, H.; Heuer, W. Highly polymerized compounds. XCIV. An insoluble polystyrene. Ber. Dtsch. Chem. Ges. B 1934, 67, 1164-1172.

(56) Gericke, M.; Schlufter, K.; Liebert, T.; Heinze, T.; Budtova, T. Rheological properties of cellulose/ionic liquid solutions: From dilute to concentrated states. Biomacromolecules 2009, 10, 1188-1194. 